OPEN ACCESS

Edited by:

Thomas Dandekar

University of Würzburg, Germany

Reviewed by:

Yajun Song,

Beijing Institute of Microbiology and Epidemiology, China

Haider Abdul-Lateef Mousa,

University of Basrah, Iraq

*Correspondence:

Liuyu Huang

huangliuyuly@163.com;

Wei Liu

liuwei5269@qq.com

tThese authors have contributed equally to this work.

Specialty section: This article was submitted to

Infectious Diseases,

a section of the journal

Frontiers in Microbiology

Received: 17 March 2016 Accepted: 27 May 2016

Published: 14 June 2016

Citation:

Jiang $X$, Dong $D$, Bian L, Zou $D$,

He $X$, Ao D, Yang Z, Huang $S$,

Liu N, Liu W and Huang L (2016) Rapid Detection of Candida albicans by Polymerase Spiral Reaction Assay

in Clinical Blood Samples.

Front. Microbiol. 7:916.

doi: 10.3389/fmicb.2016.00916

\section{Rapid Detection of Candida albicans by Polymerase Spiral Reaction Assay in Clinical Blood Samples}

\author{
Xiaoqun Jiang ${ }^{1 \dagger}$, Derong Dong ${ }^{2 \dagger}$, Lihong Bian ${ }^{3 \dagger}$, Dayang Zou ${ }^{2 \dagger}$, Xiaoming $\mathrm{He}^{2}, \mathrm{Da} \mathrm{Ao}^{2}$, \\ Zhan Yang ${ }^{2}$, Simo Huang ${ }^{2}$, Ningwei Liu ${ }^{2}$, Wei Liu' ${ }^{2 *}$ and Liuyu Huang ${ }^{2 *}$
}

\begin{abstract}
${ }^{1}$ School of Environment and Natural Resources, Renmin University of China, Beijing, China, ${ }^{2}$ Institute of Disease Control and Prevention, Academy of Military Medical Sciences, Beijing, China, ${ }^{3}$ Department of Gynecology, 307th Hospital of Chinese

People's Liberation Army, Beijing, China
\end{abstract}

Candida albicans is the most common human yeast pathogen which causes mucosal infections and invasive fungal diseases. Early detection of this pathogen is needed to guide preventative and therapeutic treatment. The aim of this study was to establish a polymerase spiral reaction (PSR) assay that rapidly and accurately detects $C$. albicans and to assess the clinical applicability of PSR-based diagnostic testing. Internal transcribed spacer 2 (ITS2), a region between 5.8S and 28S fungal ribosomal DNA, was used as the target sequence. Four primers were designed for amplification of ITS2 with the PSR method, which was evaluated using real time turbidity monitoring and visual detection using a $\mathrm{pH}$ indicator. Fourteen non-C. albicans yeast strains were negative for detection, which indicated the specificity of PSR assay was 100\%. A 10fold serial dilution of $C$. albicans genomic DNA was subjected to PSR and conventional polimerase chain reaction (PCR) to compare their sensitivities. The detection limit of PSR was $6.9 \mathrm{pg} / \mu \mathrm{l}$ within $1 \mathrm{~h}, 10$-fold higher than that of PCR $(69.0 \mathrm{pg} / \mu \mathrm{l})$. Blood samples $(n=122)$ were collected from intensive care unit and hematological patients with proven or suspected C. albicans infection at two hospitals in Beijing, China. Both PSR assay and the culture method were used to analyze the samples. Of the 122 clinical samples, 34 were identified as positive by PSR. The result was consistent with those obtained by the culture method. In conclusion, a novel and effective $C$. albicans detection assay was developed that has a great potential for clinical screening and point-of-care testing.

Keywords: C. albicans, PSR, ITS2, rapid diagnosis, isothermal

\section{INTRODUCTION}

Candida albicans is an important human yeast pathogen that accounts for the majority of superficial and systemic infections caused by the Candida genus (Fan et al., 2008; Sobel, 2015). Candidemia, the most common form of systemic candidiasis, is the fourth most frequent cause of nosocomial bloodstream infections with a mortality rate of about 50\% (Gudlaugsson et al., 2003; Kibbler et al., 2003). The morbidity of invasive candidiasis has been increasing in recent years due to the widespread use of broad-spectrum antibiotics, hormone drugs, and immunosuppressants, while invasive treatment such as endotracheal intubation and mechanically ventilation is also an important risk factor (Kim and Sudbery, 2011; Tang et al., 2016). Because Candida spp. differ in their patterns of resistance to common antifungals, differentiation of C. albicans from other 
TABLE 1 | Yeast strains included in the study.

\begin{tabular}{ll}
\hline Yeast strains & Source \\
\hline Candida albicans ATCC 24433 & Our microorganism center \\
Candida. albicans ATCC 90028 & Our microorganism center \\
C. albicans CGMCC 2.4159 & Our microorganism center \\
C. glabrata ATCC 2001 & Our microorganism center \\
C. tropicalis CGMCC 2.3967 & Our microorganism center \\
C. parapsilosis CGMCC 2.3962 & Our microorganism center \\
C. krusei CGMCC 2.1047 & Our microorganism center \\
Cryptococcus neoformans ATCC 66031 & Our microorganism center \\
C. metapsilosis ATCC 96144 & Our microorganism center \\
Saccharomyces cerevisiae CGMCC 2.3889 & Our microorganism center \\
Debaryomyces hansenii CGMCC 2.3948 & Our microorganism center \\
Kluyveromyces marxianus CGMCC 2.3959 & Our microorganism center \\
Metschnikowia pulcherrima CGMCC 2.3776 & Our microorganism center \\
Pichia membranifaciens CGMCC 2.4060 & Our microorganism center \\
P. anomala CGMCC 2.1819 & Our microorganism center \\
Kluyveromyces marxianus CGMCC 2.3959 & Our microorganism center \\
Trichosporon cutaneum CGMCC 2.2163 & Our microorganism center \\
\hline ATCC, American Type Culture Collection; CGMCC, China General Microbiological \\
Culture Collection Center. & \\
\hline
\end{tabular}

Candida species is required for appropriate preventative and antimicrobial therapy (Kim and Brehm-Stecher, 2015). Moreover, the number of clinical C. albicans isolates resistant to antifungal agents is on the rise (Liu et al., 2014). Therefore, early and accurate identification of C. albicans is critical, especially in patients with suspected symptoms of invasive candidiasis.

Conventional C. albicans detection methods based on phenotype include blood culture, microscopic examination, and biochemical identification (Bouchara et al., 1996; Alam et al., 2014). However, they are time-consuming, labor-intensive with low sensitivity. The long period of waiting time required to diagnose C. albicans infection often leads to a delay in the start of treatment with antifungal drugs. Additionally, several molecular biological methods have been applied to the detection of C. albicans, such as polimerase chain reaction (PCR; Holmes et al., 1994; Vahidnia et al., 2015), real-time PCR (Guiver et al., 2001; Maaroufi et al., 2003), mass spectrometry (Zehm et al., 2012), and immunoassay (Gunasekera et al., 2015). Nevertheless, these techniques are relatively complex and require expertise and expensive instruments. Thus, a simpler, more cost-effective method is needed.

Polymerase spiral reaction (PSR), a novel isothermal nucleic acid testing (INAT) method based on auto cycling strand displacement activity using Bst DNA polymerase, is characterized by rapidity, high specificity and high sensitivity (Liu et al., 2015). Different from PCR, which needs a strictly temperaturecontrolled and sophisticated instrument, PSR is performed under isothermal conditions. Thus, a thermostat water bath or metal bath is sufficient to initiate the PSR reaction. Compared to other established INAT techniques, PSR does not require an initial incubation at high temperature or the utilization of a DNA helicase in the reaction mix to attain the denaturation of DNA double helix (Walker et al., 1992; Notomi et al., 2000). PSR is being widely applied to the detection of bacteria, antibiotic resistance genes, and viruses (Dong et al., 2015).

Internal transcribed spacer 2 (ITS2), a region between $5.8 \mathrm{~S}$ and $28 \mathrm{~S}$ fungal ribosomal DNA (rDNA), is frequently used for identification of fungal species (Turenne et al., 1999). In this research, we designed primers targeting the ITS2 sequence and determined the specificity and sensitivity of PSR detection for C. albicans. Subsequently, the clinical applicability of the PSR method for identifying C. albicans was evaluated in intensive care unit (ICU) and hematological patients with proven or suspected candidiasis infections.

\section{MATERIALS AND METHODS}

\section{Ethics Statement}

All volunteers provided written, informed consent to participate in this study, which was reviewed and approved by the ethics committee of the Academy of Military Medical Sciences, China.

\section{Yeast Strains, Clinical Samples, and Antifungal Susceptibility Testing}

The yeast strains used in this study were listed in Table 1, with three C. albicans strains serving as the positive control. Fourteen non-C. albicans yeast strains were included as negative controls in order to evaluate the specificity of the PSR method. During the study period, 122 blood samples were collected from ICU and hematological patients with proven or suspected C. albicans infections at two comprehensive hospitals (301th hospital and 307th hospital) in Beijing, China. Besides, 10 blood samples from healthy volunteers were included as controls. The samples were analyzed by parallel examinations of PSR assay, PCR assay and conventional blood culture at independent study sites. For blood culture, briefly, all samples were evaluated in the BacT/Alert 3D blood culture system (BioMérieux, France). The positive cultures were further inoculated on blood agar plates. Species identification was conducted using the VITEK 2 automated microbiology system (BioMérieux, France) and by sequencing the ITS region of fungal rDNA (Turenne et al., 1999). Antifungal susceptibility testing was carried out using commercialized ATB FUNGUS 3 strips (BioMérieux, France). C. albicans ATCC 90028 was used as the quality-control strain.

\section{DNA Extraction}

DNA isolation from clinical blood samples was processed according to a method described by Maaroufi (Maaroufi et al., 2003). To evaluate the specificity and sensitivity of the PSR assay, C. albicans ATCC 24433 and other yeast strains were cultured in Sabouraud broth. Genomic DNA was extracted using the Dr. GenTLE (from Yeast) High Recovery kit (Takara, Japan).

\section{PSR Primer Design}

Candida albicans-specific PSR primers were designed based on the nucleotide sequence of ITS2 region obtained from the NCBI GenBank database (Accession No. KP675666.1). The nucleotide sequences of primers and their locations on ITS2 are shown in 


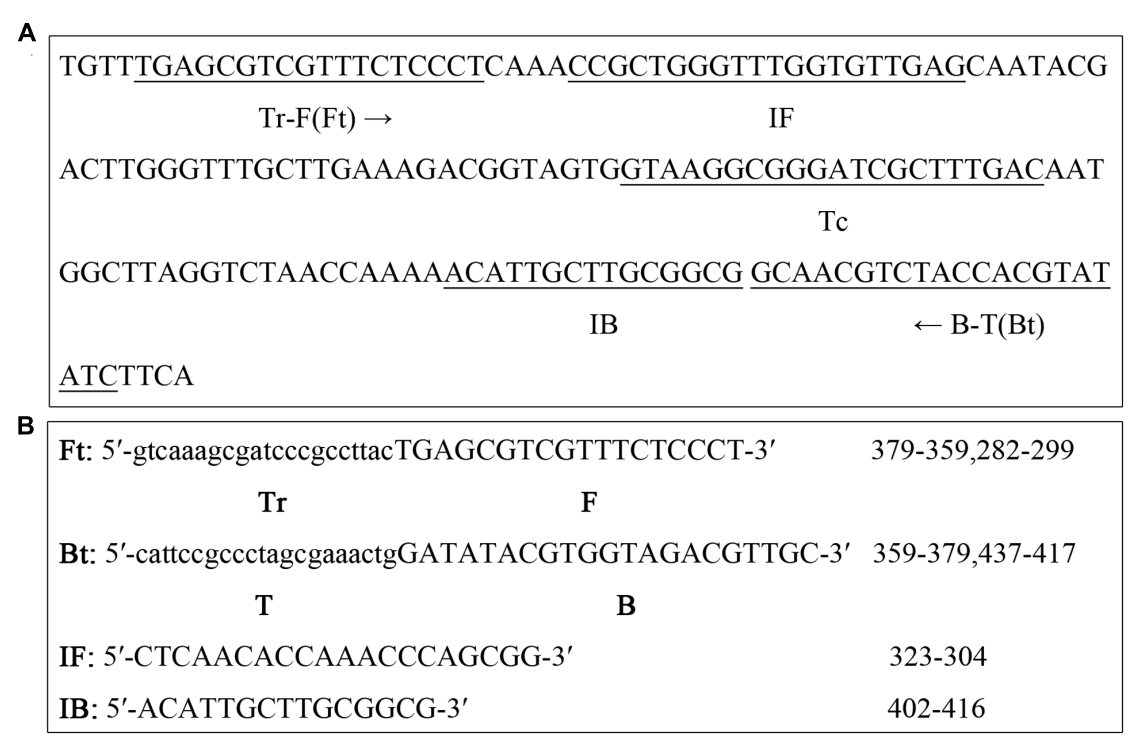

FIGURE 1 | polymerase spiral reaction (PSR) primer design for the detection of Candida albicans. (A) Locations of primers on the ITS2 region are underlined. (B) Nucleotide sequence of primers.

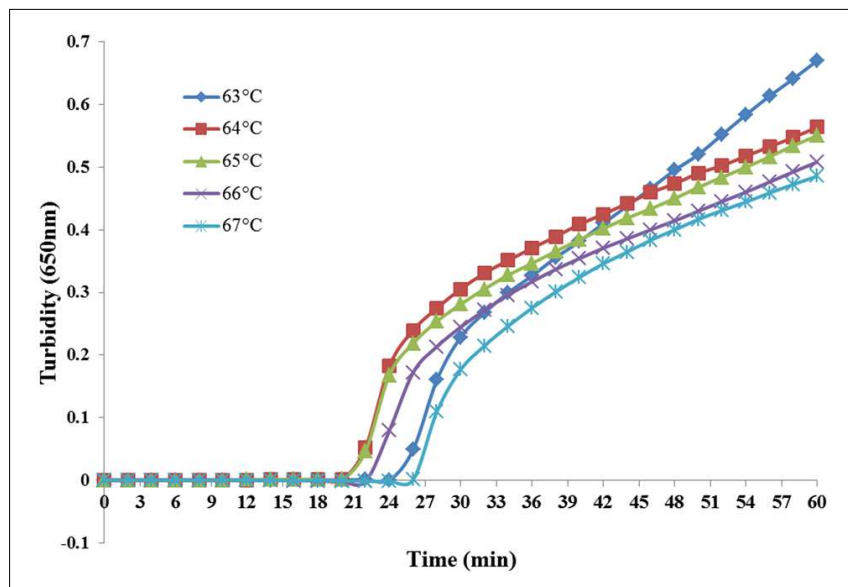

FIGURE 2 | Temperature optimization of PSR assay. Different temperatures were tested and $65^{\circ} \mathrm{C}$ was chosen as the optimal temperature for PSR amplification.

Figure 1. The uppercase sequences at the $3^{\prime}$-end of the forward primer (F) and backward primer (B) were complementary to the ITS2 region (position 282-299 and 437-417, respectively). The lowercase sequences at the $5^{\prime}$-end of the backward primer (T) were complementary to the ITS2 region (positions 359-379) and was reverse to the lowercase sequences at the $5^{\prime}$-end of the forward primer (Tr). Auxiliary primers, IF and IB (positions 323-304 and 402-416, respectively), were designed to enhance the reaction velocity.

\section{PSR Assay}

Polymerase spiral reaction reactions were carried out at $65^{\circ} \mathrm{C}$ for $60 \mathrm{~min}$ in a final volume of $25 \mu \mathrm{l}$ containing the following

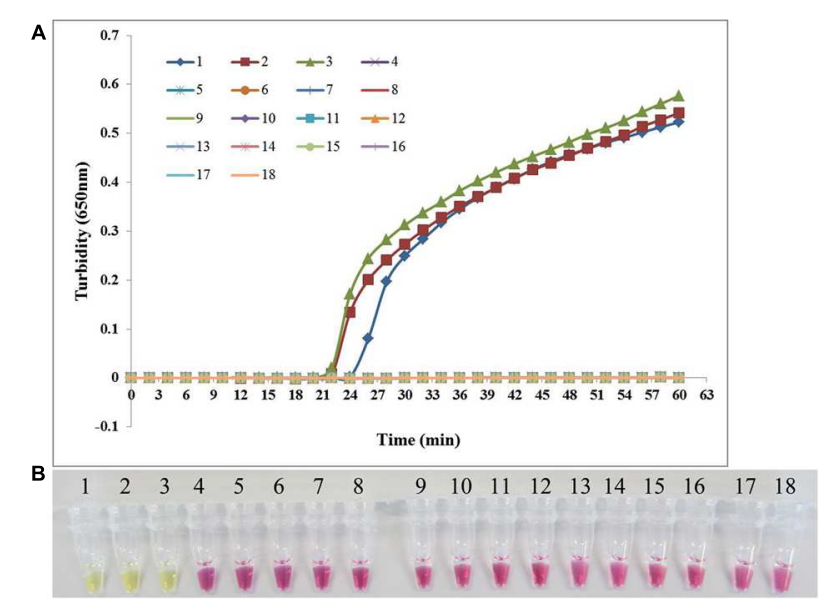

FIGURE 3 | The specificity of PSR method. The PSR assay specificity for C. albicans detection was evaluated by real-time turbidimeter (A) and the $\mathrm{pH}$ indicator colorimetric assay (B). 1, C. albicans ATCC 24433; 2, C. albicans ATCC 90028; 3, C. albicans CGMCC 2.4159; 4, Candida glabrata ATCC 2001; 5, Candida tropicalis CGMCC 2.3967; 6, Candida parapsilosis CGMCC 2.3962; 7, Candida krusei CGMCC 2.1047; 8, Cryptococcus neoformans ATCC 66031; 9, Candida metapsilosis ATCC 96144; 10, Saccharomyces cerevisiae CGMCC 2.3889; 11, Debaryomyces hansenii CGMCC 2.3948; 12 , Kluyveromyces marxianus CGMCC 2.3959; 13, Metschnikowia pulcherrima CGMCC 2.3776; 14, Pichia membranifaciens CGMCC 2.4060; 15, Pichia anomala CGMCC 2.1819; 16, Kluyveromyces marxianus CGMCC 2.3959; 17, Trichosporon cutaneum CGMCC 2.2163; 18, negative control (double-distilled water).

components: $8 \mathrm{U}$ of $B s t$ DNA polymerase; $12.5 \mu \mathrm{l}$ buffer solution (pH 8.8, containing $20 \mathrm{mM}\left(\mathrm{NH}_{4}\right)_{2} \mathrm{SO}_{4}, 100 \mathrm{mM} \mathrm{KCl}, 16 \mathrm{mM}$ $\mathrm{MgSO}_{4}, 1.6 \mathrm{M}$ betaine, $0.2 \%$ Tween 20 , and $2.8 \mathrm{mM}$ of each dNTP); and $2.0 \mu \mathrm{l}$ DNA template. The amount of primers per 


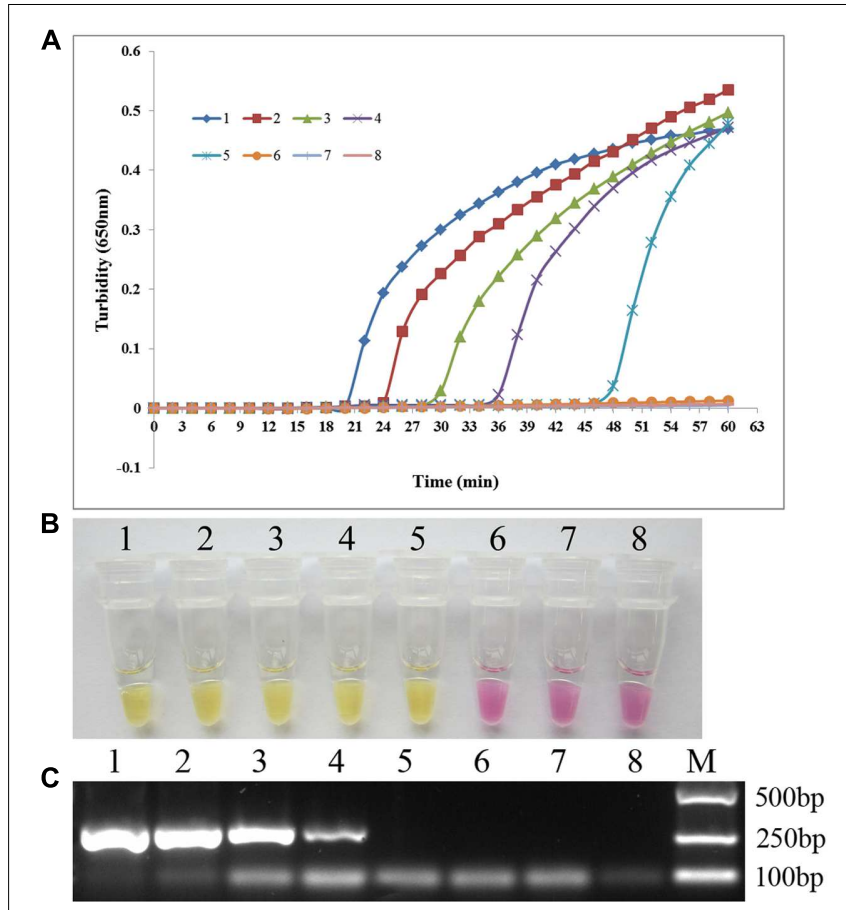

FIGURE 4 | The sensitivity of PSR assay. Sensitivity of the PSR method (A, B) versus conventional PCR (C) for the detection of C. albicans. 1-7, 10-fold serial dilutions of $C$. albicans ATCC 24433 genomic DNA (69.0 ng/ $/ \mathrm{l}-0.069 \mathrm{pg} / \mu \mathrm{l}) ;$ 8, double-distilled water.

reaction was $0.8 \mu \mathrm{M}$ for IF and IB and $1.6 \mu \mathrm{M}$ for Ft and Bt. Finally, the reaction mixture was covered with a protectant (patent: ZL201210371448.5, China) to prevent aerosol crosscontamination.

Amplification was monitored by two methods, turbidity monitoring with a real-time turbidimeter (LA-320c; Eiken Chemical, Japan) and direct visual in the presence of $\mathrm{pH}$ dye. A $1-\mu l$ volume of $\mathrm{pH}$ indicator (containing cresol red, $2.0 \mathrm{mM}$; phenol red, $0.6 \mathrm{mM}$ ) was added to the reaction tube; the color change from red to yellow for positive samples was visible by naked eye under natural light, while the negative reaction stayed red (Tanner et al., 2015). Each experiment was repeated at least three times.

\section{PCR Assay}

The PCR method was conducted using primers SAP-F $\left(5^{\prime}\right.$ - CTGCTGATATTACTGTTGGTTC- $\left.3^{\prime}\right)$ and SAP-B (5'CCACCAATACCAACGGTATC- $\left.3^{\prime}\right)$. The reaction conditions were in accordance with the previously published papers (Flahaut et al., 1998). The expected PCR product size was $263 \mathrm{bp}$. The products were separated by electrophoresis on a $1 \%$ agarose gel
TABLE 3 | Results of antifungal susceptibility testing of the 34 C. albicans isolates.

\begin{tabular}{lccc}
\hline Antifungal drug & Sensitive (\%) & Intermediate (\%) & Resistant (\%) \\
\hline 5-Fluorocytosine & 91.2 & 0 & 8.8 \\
Fluconazole & 79.4 & 5.9 & 14.7 \\
Voriconazole & 70.6 & 11.8 & 17.6 \\
Itraconazole & 82.4 & 11.7 & 5.9 \\
Amphotericin B & 100 & 0 & 0
\end{tabular}

(Amresco, USA), which was stained by GelRed (Biotium, USA) and imaged using the Gel Doc XR+ system (Bio-Rad, USA).

\section{RESULTS}

\section{Temperature Optimization of PSR Assay}

Reaction temperatures ranging from 63 to $67^{\circ} \mathrm{C}$ at $1^{\circ} \mathrm{C}$ increments were compared for optimal amplification. As shown in Figure 2, both 64 and $65^{\circ} \mathrm{C}$ sets amplified the target sequence within the shortest time. However, $65^{\circ} \mathrm{C}$ is the optimal temperature for the enzymatic activity of Bst DNA polymerase (Aliotta et al., 1996) and was, therefore, chosen for the following experiments.

\section{Specificity of the PSR Assay}

Candida albicans ATCC 24433, C. albicans ATCC 90028, and C. albicans CGMCC 2.4159 were used as the positive control and double-distilled water as the blank control when assessing the specificity of PSR method for the detection of C. albicans. In addition, 14 non- $C$. albicans yeast strains were tested. As shown in Figure 3, both real-time turbidity monitoring and visual detection correctly identified C. albicans. All other yeast strains tested negative in addition to the blank control, indicating that PSR assay can detect C. albicans with high specificity.

\section{Comparison between the Sensitivities of PSR and PCR for C. albicans Detection}

To compare the sensitivities of the PSR method and conventional PCR amplification, we conducted 10-fold serial dilutions of C. albicans ATCC 24433 genomic DNA $(69.0 \mathrm{ng} / \mu \mathrm{l}-0.069 \mathrm{pg} / \mu \mathrm{l})$ and subjected them to sensitivity testing. The detection limit for PSR was $6.9 \mathrm{pg} / \mu \mathrm{l}$ based on either turbidity measurement or visual inspection, 10-fold higher than that of conventional PCR (Figure 4). The results indicated that PSR assay is highly sensitive for the detection of C. albicans.

\section{Clinical Application of the PSR Method}

To assess the clinical applicability of the PSR method, we analyzed 122 blood samples from patients with proven or suspected

TABLE 2 | Comparison of the results obtained from clinical blood samples by the method of PSR, PCR and blood culture.

\begin{tabular}{|c|c|c|c|c|c|c|}
\hline & Total & Positive by culture & False negative by PSR & False positive by PSR & False negative by PCR & False positive by PCR \\
\hline No. of samples & 132 & 34 & 0 & 0 & 2 & 0 \\
\hline
\end{tabular}


candidiasis infection by PSR, PCR and conventional blood culture. Thirty-four samples were tested positive for C. albicans by PSR (Table 2), in agreement with the culture method. There were false-negative results by PCR assay with two samples. None of the control samples from healthy volunteers was positive in these methods. Sequence analyses of the ITS2 region from the 34 C. albicans isolates was conserved with the nucleotide sequences reported previously. Antifungal susceptibility testing was carried out to further characterize the clinical C. albicans isolates. The susceptibility rate was $91.2 \%$ for 5 -fluorocytosine, $79.4 \%$ for fluconazole, $70.6 \%$ for voriconazole, and $82.4 \%$ for itraconazole; none of the tested strains showed resistance to amphotericin $B$ (Table 3).

\section{DISCUSSION}

Normally living as a harmless commensal, C. albicans commonly resides in mucosal surfaces of the gastrointestinal and genitourinary tracts (Zucchi et al., 2010). However, overgrowth of $C$. albicans will result in mucosal and superficial diseases such as oral candidiasis and vulvo vaginal candidiasis (VVC). Moreover, C. albicans can enter the systemic circulation through epithelial tissue, causing critical invasive candidiasis such as bloodstream infections, bronchopneumonia, and meningitis. Unfortunately, early diagnosis of invasive candidiasis remains a challenge because of unapparent symptoms (e.g., a prolonged low-grade fever) and time-consuming fungal culture methods (Tang et al., 2016). Thus, early and accurate identification of C. albicans is very important.

The development of INAT methods has greatly facilitated clinical screening and on-site diagnosis ( $\mathrm{Li}$ and Macdonald, 2015). In this study, the PSR method, a recently developed INAT technique, was used to detect $C$. albicans targeting the ITS2 gene. Additionally, two auxiliary primers were designed to improve the reaction velocity. When the Bst DNA polymerase incorporates a deoxynucleoside triphosphate into the nascent DNA, the byproducts include a hydrogen ion (Pourmand et al., 2006). The accumulation of hydrogen ions results in the decrease of $\mathrm{pH}$ value, which enabled the use of $\mathrm{pH}$ indicator. Both continuous monitoring with a real-time turbidimeter and visual inspection with the aid of cresol red and phenol red were used to assess the PSR amplification of the ITS2 gene, with an identical sensitivity of $6.9 \mathrm{pg} / \mu \mathrm{l}$ within $60 \mathrm{~min}$, which was 10 times more sensitive than traditional PCR $(69.0 \mathrm{pg} / \mu \mathrm{l})$. Moreover, no false-positive amplification was observed when testing non-C. albicans species.

The PSR method requires a constant-temperature environment, so a thermostatic water bath or even a vacuum

\section{REFERENCES}

Alam, M. Z., Alam, Q., Jiman-Fatani, A., Kamal, M. A., Abuzenadah, A. M., Chaudhary, A. G., et al. (2014). Candida identification: a journey from conventional to molecular methods in medical mycology. World J. Microbiol. Biotechnol. 30, 1437-1451. doi: 10.1007/s11274-0131574-z

Aliotta, J. M., Pelletier, J. J., Ware, J. L., Moran, L. S., Benner, J. S., and Kong, H. (1996). Thermostable Bst DNA polymerase I lacks a cup is sufficient to perform the PSR reaction. In contrast, PCR involves temperature cycling. It is also worth mentioning that covering the reaction mixture with a wax seal was essential for minimizing cross contamination, without disturbing the PSR reaction.

The clinical applicability of PSR was confirmed through a surveillance of $C$. albicans in blood samples. The test results indicated that $34 / 122$ of samples tested positive, which was in coincidence with the culture method. However, the latter requires at least 2-3 days, while it takes less than $60 \mathrm{~min}$ for PSR-based detection. There were two false-negative results by PCR assay (sensitivity: 94.1\%), indicating PSR is more sensitive than PCR for detection of samples with low C. albicans loads. In addition, the PSR method is easy to operate and obviates the need for complicated instruments. It is fast and convenient to identify the result as positive or negative through color change. Thus, we think PSR has a great potential for clinical screening. The antifungal susceptibility testing of $C$. albicans isolates revealed that amphotericin $\mathrm{B}$ may be beneficial when azole antifungal agents are ineffective for VVC treatment.

\section{CONCLUSION}

We established a novel PSR detection method for C. albicans, which meets the guidelines proposed by the World Health Organization for developing diagnostic techniques-namely, ASSURED (affordable, sensitive, specific, user-friendly, robust and rapid, equipment-free, and deliverable; Mabey et al., 2004). We anticipate its routine use for on-site testing in clinical settings, especially in situations where resources are limited.

\section{AUTHOR CONTRIBUTIONS}

DD, DZ, XH, and DA did the experiment. XJ and LB provided the clinical samples. $\mathrm{ZY}$ and $\mathrm{SH}$ designed the experiments. NL analyzed the data. WL and LH managed the project and wrote the article.

\section{FUNDING}

This work was supported by the Mega-projects of Science and Technology Research of China (Grant 2013ZX10004-203) and the National Natural Science Foundation of China (Grant No. 81501836).

$3^{\prime}->5^{\prime}$ proofreading exonuclease activity. Genet. Anal. 12, 185-195. doi: 10.1016/S1050-3862(96)80005-2

Bouchara, J. P., Declerck, P., Cimon, B., Planchenault, C., de Gentile, L., and Chabasse, D. (1996). Routine use of CHROMagar Candida medium for presumptive identification of Candida yeast species and detection of mixed fungal populations. Clin. Microbiol. Infect. 2, 202-208. doi: 10.1016/S1198743X(14)65143-0

Dong, D., Zou, D., Liu, H., Yang, Z., Huang, S., Liu, N., et al. (2015). Rapid detection of Pseudomonas aeruginosa targeting the toxA gene in 
intensive care unit patients from Beijing, China. Front. Microbiol. 6:1100. doi: 10.3389/fmicb. 2015.01100

Fan, S. R., Bai, F. Y., Liao, Q. P., Liu, Z. H., Li, J., and Liu, X. P. (2008). Genotype distribution of Candida albicans strains associated with different conditions of vulvovaginal candidiasis, as revealed by microsatellite typing. Sex. Transm. Infect. 84, 103-106. doi: 10.1136/sti.2007. 025700

Flahaut, M., Sanglard, D., Monod, M., Bille, J., and Rossier, M. (1998). Rapid detection of Candida albicans in clinical samples by DNA amplification of common regions from C. albicans-secreted aspartic proteinase genes. J. Clin. Microbiol. 36, 395-401.

Gudlaugsson, O., Gillespie, S., Lee, K., Vande, Berg, J., Hu, J., Messer, S., et al. (2003). Attributable mortality of nosocomial candidemia, revisited. Clin. Infect. Dis. 37, 1172-1177. doi: 10.1086/378745

Guiver, M., Levi, K., and Oppenheim, B. A. (2001). Rapid identification of candida species by TaqMan PCR. J. Clin. Pathol. 54, 362-366. doi: 10.1136/jcp.54. 5.362

Gunasekera, M., Narine, M., Ashton, M., and Esfandiari, J. (2015). Development of a Dual Path Platform (DPP ${ }^{\circledR}$ ) immunoassay for rapid detection of Candida albicans in human whole blood and serum. J. Immunol. Methods 424, 7-13. doi: 10.1016/j.jim.2015.04.014

Holmes, A. R., Cannon, R. D., Shepherd, M. G., and Jenkinson, H. F. (1994). Detection of Candida albicans and other yeasts in blood by PCR. J. Clin. Microbiol. 32, 228-231.

Kibbler, C. C., Seaton, S., Barnes, R. A., Gransden, W. R., Holliman, R. E., Johnson, E. M., et al. (2003). Management and outcome of bloodstream infections due to Candida species in England and Wales. J. Hosp. Infect. 54, 18-24. doi: 10.1016/S0195-6701(03)00085-9

Kim, H. J., and Brehm-Stecher, B. F. (2015). Design and evaluation of peptide nucleic acid probes for specific identification of Candida albicans. J. Clin. Microbiol. 53, 511-521. doi: 10.1128/JCM.02417-14

Kim, J., and Sudbery, P. (2011). Candida albicans, a major human fungal pathogen. J. Microbiol. 49, 171-177. doi: 10.1007/s12275-011-1064-7

Li, J., and Macdonald, J. (2015). Advances in isothermal amplification: novel strategies inspired by biological processes. Biosens. Bioelectron. 15, 196-211. doi: 10.1016/j.bios.2014.08.069

Liu, S., Hou, Y., Chen, X., Gao, Y., Li, H., and Sun, S. (2014). Combination of fluconazole with non-antifungal agents: a promising approach to cope with resistant Candida albicans infections and insight into new antifungal agent discovery. Int. J. Antimicrob. Agents 43, 395-402. doi: 10.1016/j.ijantimicag.2013.12.009

Liu, W., Dong, D., Yang, Z., Zou, D., Chen, Z., Yuan, J., et al. (2015). Polymerase spiral reaction (PSR): a novel isothermal nucleic acid amplification method. Sci. Rep. 5, 12723. doi: 10.1038/srep12723

Maaroufi, Y., Heymans, C., De Bruyne, J. M., Duchateau, V., RodriguezVillalobos, H., Aoun, M., et al. (2003). Rapid detection of Candida albicans in clinical blood samples by using a TaqMan-based PCR assay. J. Clin. Microbiol. 41, 3293-3298. doi: 10.1128/JCM.41.7.3293-3298. 2003
Mabey, D., Peeling, R. W., Ustianowski, A., and Perkins, M. D. (2004). Diagnostics for the developing world. Na., Rev. Microbiol. 2, 231-240. doi: 10.1038/nrmicro841

Notomi, T., Okayama, H., Masubuchi, H., Yonekawa, T., Watanabe, K., Amino, N., et al. (2000). Loop-mediated isothermal amplification of DNA. Nucleic Acids Res. 28, E63. doi: 10.1093/nar/28.12.e63

Pourmand, N., Karhanek, M., Persson, H. H., Webb, C. D., Lee, T. H., Zahradníková, A., et al. (2006). Direct electrical detection of DNA synthesis. Proc. Natl. Acad. Sci. U.S.A. 103, 6466-6470. doi: 10.1073/pnas.06011 84103

Sobel, J. D. (2015). Recurrent vulvovaginal candidiasis. Am. J. Obstet. Gynecol. 214, 15-21. doi: 10.1016/j.ajog.2015.06.067

Tang, X. L., Hua, Y., Guan, Q., and Yuan, C. H. (2016). Improved detection of deeply invasive candidiasis with DNA aptamers specific binding to $(1 \rightarrow 3)-\beta-D$ glucans from Candida albicans. Eur. J. Clin. Microbiol. Infect. Dis. 35, 587-595. doi: 10.1007/s10096-015-2574-8

Tanner, N. A., Zhang, Y., and Evans, T. C. Jr. (2015). Visual detection of isothermal nucleic acid amplification using $\mathrm{pH}$-sensitive dyes. Biotechniques 58, 59-68. doi: 10.2144/000114253

Turenne, C. Y., Sanche, S. E., Hoban, D. J., Karlowsky, J. A., and Kabani, A. M. (1999). Rapid identification of fungi by using the ITS2 genetic region and an automated fluorescent capillary electrophoresis system. J. Clin. Microbiol. 37, 1846-1851.

Vahidnia, A., Bekers, W., Bliekendaal, H., and Spaargaren, J. (2015). High throughput multiplex-PCR for direct detection and diagnosis of dermatophyte species, Candida albicans and Candida parapsilosis in clinical specimen. J. Microbiol. Methods 113, 38-40. doi: 10.1016/j.mimet.2015.03.024

Walker, G. T., Fraiser, M. S., Schram, J. L., Little, M. C., Nadeau, J. G., and Malinowski, D. P. (1992). Strand displacement amplification-an isothermal, in vitro DNA amplification technique. Nucleic Acids Res. 11, 1691-1696. doi: 10.1093/nar/20.7.1691

Zehm, S., Schweinitz, S., Würzner, R., Colvin, H. P., and Rieder, J. (2012). Detection of Candida albicans by mass spectrometric fingerprinting. Curr. Microbiol. 64, 271-275. doi: 10.1007/s00284-011-0064-5

Zucchi, P. C., Davis, T. R., and Kumamoto, C. A. (2010). A Candida albicans cell wall-linked protein promotes invasive filamentation into semi-solid medium. Mol. Microbiol. 76, 733-748. doi: 10.1111/j.1365-2958.2010.07137.x

Conflict of Interest Statement: The authors declare that the research was conducted in the absence of any commercial or financial relationships that could be construed as a potential conflict of interest.

Copyright (c) 2016 Jiang, Dong, Bian, Zou, He, Ao, Yang, Huang, Liu, Liu and Huang. This is an open-access article distributed under the terms of the Creative Commons Attribution License (CC BY). The use, distribution or reproduction in other forums is permitted, provided the original author(s) or licensor are credited and that the original publication in this journal is cited, in accordance with accepted academic practice. No use, distribution or reproduction is permitted which does not comply with these terms. 